\title{
Cardiac and gingival metastasis after total abdominal hysterectomy with bilateral salpingo-oophorectomy for primary uterine epithelioid angiosarcoma: case report and review of the literature
}

\author{
Olivier Donnez $\cdot$ Etienne Marbaix • \\ Patrick Van Ruyssevelt • Sarah Mitri • Jacques Donnez
}

Received: 12 June 2009 /Accepted: 13 August 2009/Published online: 15 September 2009

(C) Springer-Verlag 2009

\begin{abstract}
Uterine epithelioid angiosarcomas are extremely rare; only 24 cases have been documented worldwide. We present a unique case of cardiac and gingival metastases developing 4 years after total abdominal hysterectomy with bilateral salpingo-oophorectomy for primary uterine epithelial angiosarcoma. Initial treatment remains total abdominal hysterectomy with bilateral salpingo-oophorectomy. Limited distant metastases may be surgically resected in selected cases in order to improve quality of life or to prevent sudden death in untreated patients. Optimal chemotherapy regimens must be determined.
\end{abstract}

Keywords Primary uterine angiosarcoma $\cdot$ Cardiac metastasis · Gingival metastasis

\section{O. Donnez}

Department of Gynecology,

Cliniques Universitaires de Mont-Godinne,

5530 Yvoir, Belgium

\section{E. Marbaix}

Department of Anatomopathology,

Université Catholique de Louvain,

1200 Brussels, Belgium

P. Van Ruyssevelt

Cardiovascular Department,

Centre Hospitalier de Jolimont-Lobbes,

7160 Haine-Saint-Paul, Belgium

S. Mitri $\cdot$ J. Donnez $(\triangle)$

Department of Gynecology, Université Catholique de Louvain, Cliniques Universitaires St Luc,

Avenue Hippocrate 10,

1200 Brussels, Belgium

e-mail: jacques.donnez@uclouvain.be

\section{Introduction}

Epithelioid angiosarcoma was first described in 1864 by Klob [1]. It can arise from any blood or lymph vessels and most commonly occurs on the face and scalp and is linked to chronic lymphedema and previous irradiation [2]. It accounts for less than $2 \%$ of all sarcomas [3]. In the uterus, epithelioid angiosarcomas are extremely rare; only 24 cases have been documented worldwide $[4,5]$.

Metastases of uterine epithelioid angiosarcomas are poorly described in the literature. Here, we report the first case of cardiac metastasis arising from epithelioid angiosarcoma of the uterus 4 years after total abdominal hysterectomy with bilateral salpingo-oophorectomy. Successful resection of gingival metastasis is also reported.

\section{Case report}

The patient's oncological history dates back to a very early age. At just 2.5 years of age, she underwent surgical resection of a liposarcoma from the hip. Local recurrence was observed 1.5 years later, and surgical resection was followed by external radiotherapy. No metastatic lesions were identified. Unfortunately, no information is available on the timing or administered doses.

The patient then had an unremarkable medical history until the age of 58, when she presented with chronic cystitis and progressive abdominal discomfort. A large uterine tumor was diagnosed. Because of the considerable volume of the initial tumor, she underwent total abdominal hysterectomy and bilateral salpingo-oophorectomy. The uterine weight was $2,500 \mathrm{~g}$. Histological examination of the uterus revealed a normal endometrium and normal 
adnexa on both sides. The uterine corpus was the site of the primary tumor. Histology, shown in Fig. 1a (low power magnification) and $b$ (high power magnification), revealed irregular anastomotic vascular channels. Neoplastic cells had a pleomorphic vesicular nucleus with a prominent nucleolus and showed mitotic activity. The lesion was identified as an epithelioid angiosarcoma of the uterus. CD31 (Fig. 1c) was strongly immunolabeled in all neoplastic cells and CD34 (Fig. 1d) in most neoplastic cells, while von Willebrand factor (Fig. 1e) was only faintly detected in some neoplastic cells. Cytokeratin (Fig. 1f) was immunolabeled in many neoplastic cells using the AE1AE3 antibody mixture.

Bone metastasis in L2 and pulmonary lesions were also detected, and the patient underwent palliative adjuvant chemotherapy based on ifosfamide and doxorubicin (four regimens) associated with zoledronic acid. After two regimens, she presented with periodontal ulceration of the
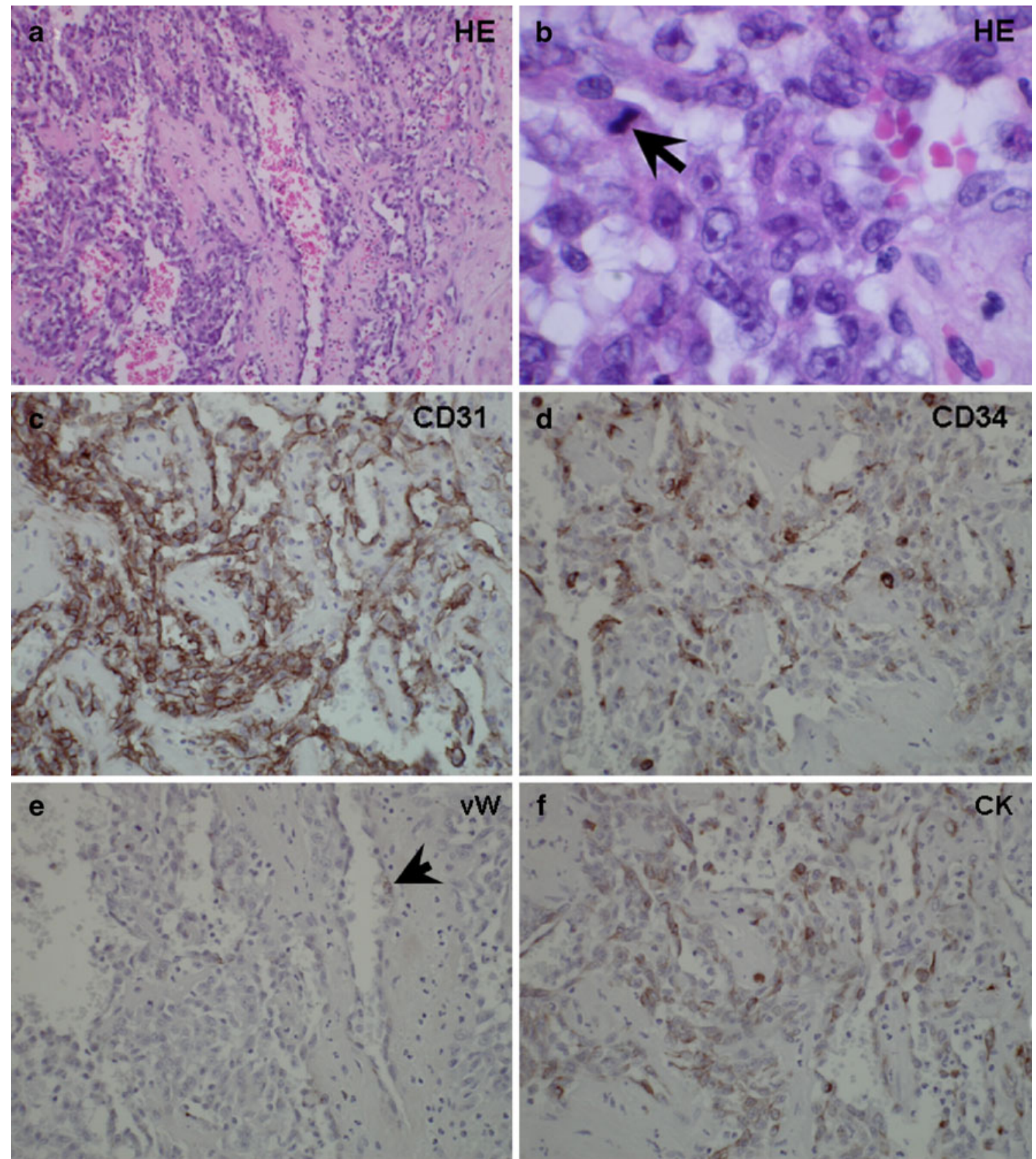

Fig. 1 a Low and $\mathbf{b}$ high power magnifications of the primary tumor in the uterine corpus reveal irregular anastomotic vascular channels. Neoplastic cells have a pleomorphic vesicular nucleus with a prominent nucleolus and show mitotic activity (black arrow). c CD31 is strongly

immunolabeled in all neoplastic cells. d CD34 is immunolabeled in most neoplastic cells. e von Willebrand factor is faintly detected in some neoplastic cells (black arrow). f Cytokeratin is immunolabeled in many neoplastic cells using the AE1-AE3 antibody mixture 
upper maxilla. Upon completion of the chemotherapy, the bone and pulmonary metastases were found to have regressed, but not the upper maxillary ulceration. The patient, therefore, underwent surgical resection of the maxilla, and histopathological examination (Fig. 2) revealed an angiosarcomatous growth beneath the squamous epithelium at the edge of the ulcer. One and a half years after the end of chemotherapy, chest and abdominal tomography confirmed the absence of recurrence. Bone scintigraphy showed stability of the lumbar spine, particularly L2, over a period of 4 years.

At the age of 62, the patient presented with hyperthermic syndrome associated with hyperleukocytosis $(22,000 / \mu \mathrm{l})$. A positron emission tomography scan revealed hyperfixation at the level of the superior pole of the left kidney and right cardiac ventricle. Cardiac echography was normal, and left adrenalectomy was performed. Histopathological examination did not evidence any neoplastic lesions, so immunohistochemical analysis was not carried out. The patient then received corticotherapy, and close follow-up was proposed. Two weeks later, she presented with dyspnea and distal cyanosis, and pulmonary scintigraphy showed bilateral embolism. Thoracic tomography revealed a mass inside the right atrium. Cardiac echography confirmed the presence of a $45-\mathrm{mm}$ mass growing from the right atrial wall and obtruding the superior vena cava.

Because of the persistence of normal cardiac function and rapid development of the lesion, excisional surgery was proposed. After open heart surgery, a necrotic mass measuring $45 \mathrm{~mm}$ was observed issuing from the right wall of the right auricle (Fig. 3). En bloc excision and complete reconstruction of the right atrium using equine pericardium were performed. Histopathological findings (Fig. 4) showed endocardial aggregates of proliferating

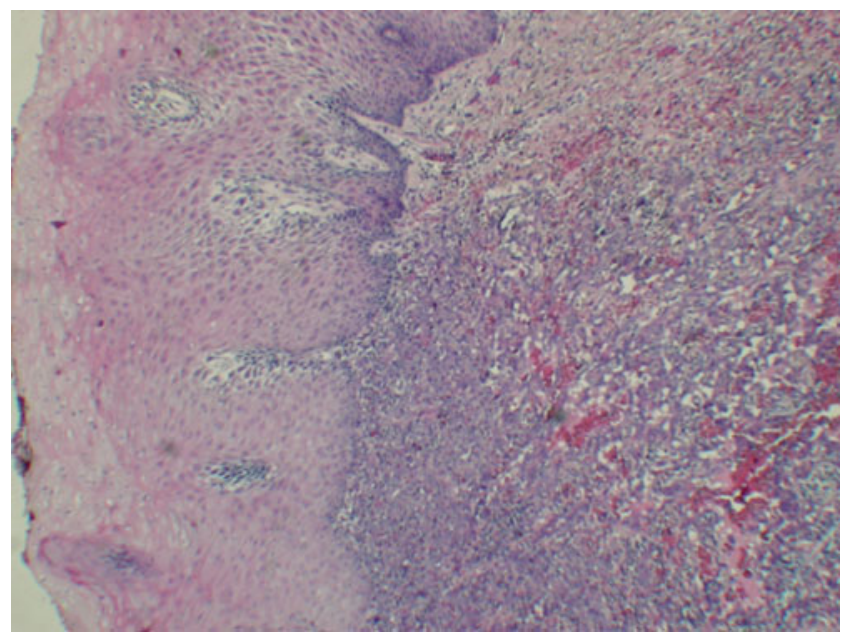

Fig. 2 Palatal metastasis shows an angiosarcomatous growth beneath the squamous epithelium at the edge of the ulcer

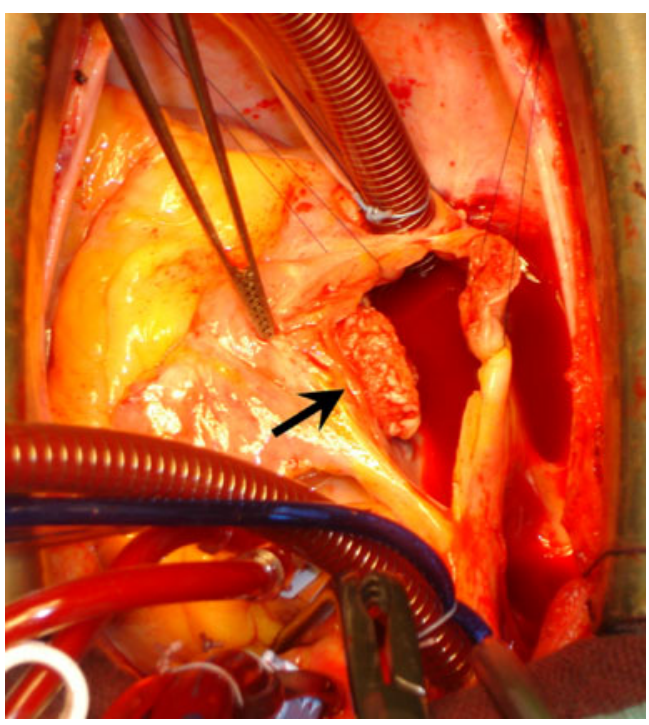

Fig. 3 Open heart surgery reveals a $4.5-\mathrm{cm}$ mass in the right atrial wall (black arrow)

pleomorphic cells with central necrosis and confirmed the presence of cardiac metastasis of angiosarcomatous origin.

Unfortunately, during close postoperative follow-up, the patient developed acute severe right cardiac failure, complicated by refractory venous stasis and liver and kidney failures, and subsequently died. A recurrent pulmonary embolism was suspected.

\section{Discussion}

The 5-year relative survival rate for all uterine sarcomas is $43.5 \%$ (95\% confidence intervals: $42.0-44.9$ ) [6]. Uterine sarcomas other than leiomyosarcomas and endometrial

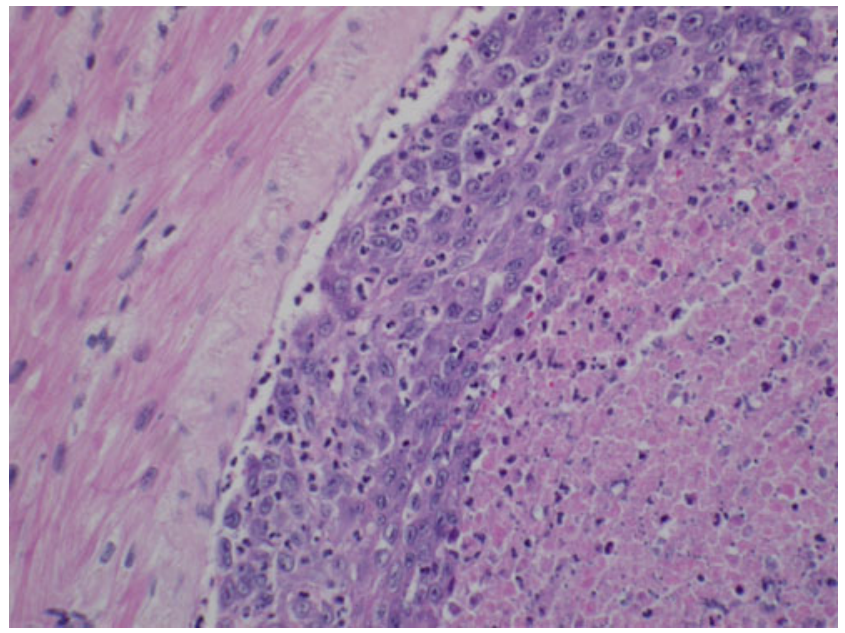

Fig. 4 Cardiac metastasis comprises endocardial aggregates of proliferating pleomorphic cells with central necrosis 
stromal sarcomas are exceedingly rare. According to Olawaiye et al. [4] and Cardinale et al. [5], only 24 cases of uterine angiosarcoma have ever been published in the English-speaking literature. Since routine immunohistochemical investigation was introduced in the early 1980s, only 15 cases $[4,5,7-15]$ have been documented using this technique in addition to morphological analysis. In the case we describe, angiosarcoma was microscopically identified by the freely anastomosing vascular channels, but also by positive immunohistochemical staining for endothelial cell antigens such as CD31, CD34, and von Willebrand factor. The same observations were made of both the gingival and cardiac metastases.

In Table 1, we present the last 16 cases of primary uterine angiosarcoma diagnosed by morphology and immunohistochemistry. We deliberately did not take into account cases reported before the early 1980s because of the absence of routine immunohistochemistry [7]. Ninety-three percent $(n=15 / 16)$ of patients were peri- or postmenopausal at the time of diagnosis. One patient was only 35 years of age [5], but no data are available on her evolution after surgery. In this case, the prognosis was very poor due to the reported presence of malignant cells in omental nodules and cytology fluid smears from pelvic irrigation and pleural effusions.

According to the literature, the prognosis of uterine angiosarcomas is very poor, with a median survival of just 13.5 months (Table 1). Small tumors $[9,12]$ appear to have a better prognosis. This view is upheld by Schammel and Tavassoli [12], who strongly believe that the endocavitary growth pattern might be considered a favorable prognostic factor. They previously reported a case of a patient still alive more than 3 years after a diagnosis of angiosarcoma, which is the only case of uterine angiosarcoma exhibiting an exophytic polyp rather than diffuse neoplastic infiltration of the myometrium. This is not supported by our findings, however. Our patient presented with diffuse myometrial infiltration of a 2,500-g uterus, and she survived for more than 4 years. Since we have no further information about the evolution of the cases described by Quinonez et al. [9] ( $>47$ months' survival at the time of the case report) and Schammel et al. [12] (>36 months' survival at the time of the case report), our patient, having survived for 53 months, shows the longest overall survival observed after treatment of primary uterine angiosarcoma to date. This may have

Table 1 Sixteen cases of primary uterine angiosarcoma diagnosed by morphology and immunohistochemistry

\begin{tabular}{|c|c|c|c|c|c|c|c|c|}
\hline Year & Age & Macroscopic features & Surgery & $\begin{array}{l}\text { Adjuvant } \\
\text { chemotherapy }\end{array}$ & $\begin{array}{l}\text { Adjuvant } \\
\text { irradiation }\end{array}$ & $\begin{array}{l}\text { PFS } \\
\text { months }\end{array}$ & $\begin{array}{l}\text { OS } \\
\text { months }\end{array}$ & Metastasis \\
\hline 1987 & 71 & $\begin{array}{l}\text { Massive enlargement } \\
\text { of uterus }\end{array}$ & TAH, BSO & None & Yes & 3 & 3 & NA \\
\hline 1990 & 76 & $\begin{array}{l}\text { Massive enlargement } \\
\text { of uterus }\end{array}$ & TAH, BSO & None & None & 6 & 6 & NA \\
\hline 1991 & 65 & $\begin{array}{l}\text { 4.7-cm hemorr } \\
\text { mass + bulky uterus }\end{array}$ & TAH, BSO LN & Cisplatin + adriamycin & Yes & 47 & 47 & NA \\
\hline 1993 & 56 & $\begin{array}{l}\text { Multiple hemorr } \\
\text { myoma }\end{array}$ & TAH, BSO LN & Ifosfamide + adriamycin & Yes & 2 & 7 & NA \\
\hline 1993 & 61 & 10 -cm uterine mass & TAH, BSO & None & None & 1 & 1 & NA \\
\hline 1994 & 58 & 12-cm mass & TAH, BSO & Unspecified type & Yes & 2 & 2 & NA \\
\hline \multirow[t]{4}{*}{1998} & 49 & 6.3-kg uterine mass & TAH, BSO & None & None & 3 & 3 & NA \\
\hline & 58 & 12-cm uterine mass & TAH, BSO & Unspecified type & Yes & 2 & 2 & NA \\
\hline & 70 & $\begin{array}{l}\text { 5-cm polypoid lesion, } \\
\text { half myoma }\end{array}$ & TAH, BSO & None & None & 36 & 36 & NA \\
\hline & 75 & $6.3-\mathrm{cm}$ lower uterus & TAH, BSO & None & None & 7 & 7 & NA \\
\hline 1999 & 59 & 12-week size uterus & TAH, BSO LN & None & None & 0.4 & 2.5 & Vaginal, lung, brain \\
\hline 2001 & 67 & $25 \times 21-\mathrm{cm}$ uterus & TAH, BSO & None & None & 0 & 15 & Gingival, lung, brain \\
\hline \multirow[t]{2}{*}{2008} & 81 & 200-g uterus & TAH, BSO & None & None & 0 & 6 & Peritoneal spread \\
\hline & 35 & $2.400-\mathrm{g}$ uterus & TAH, BSO & NA & NA & 0 & NA & $\begin{array}{l}\text { Peritoneal+pleural } \\
\text { spread }\end{array}$ \\
\hline \multirow[t]{2}{*}{2008} & 54 & $11-\mathrm{cm}$ bulky uterus & TAH, BSO & Gemcitabine + taxotere & None & 3 & 12 & $\begin{array}{l}\text { Peritoneal }+ \text { pleural } \\
\text { spread }\end{array}$ \\
\hline & & & & Bevacizumab & & & & \\
\hline 2009 & 58 & $2.500-\mathrm{g}$ uterus & TAH, BSO & Ifosfamide + adriamycin & None & 3 & 53 & $\begin{array}{l}\text { Bone, lung, gingival, } \\
\text { heart }\end{array}$ \\
\hline
\end{tabular}

$T A H$ total abdominal hysterectomy, BSO bilateral salpingo-oophorectomy, $L N$ pelvic lymphadenectomy, $N A$ not applicable 
been due to the chemotherapy administered, but no other data are available in the literature on the impact of four regimens of ifosfamide and doxorubicin.

Complete surgical resection is generally accepted as the primary treatment in the literature. Olawaiye et al. [4] recommend total abdominal hysterectomy with bilateral salpingo-oophorectomy, as well as surgical excision of all macroscopic lesions observed in the peritoneal cavity. There are no data supporting routine pelvic or para-aortic lymphadectomy at the time of surgery. Indeed, no lymph node involvement has ever been reported, even in case of extensive peritoneal spread $[9,10,14]$. This is corroborated by histological findings, which show a strong tendency toward proliferation by hematogenous metastasis.

Olawaiye et al. [4] report that adjuvant chemotherapy may be of benefit in case of uterine angiosarcoma. Of patients (Table 1) who received adjuvant chemotherapy, $50 \%(n=3 / 6)$ were still alive more than 12 months after treatment (Table 1). Of those who did not receive chemotherapy, only $11 \%(n=1 / 9)$ survived after treatment. Moreover, the surviving patient was reported to have an angiosarcoma in a 5-cm intrauterine polyp, without transmural myometrial invasion [12]. These authors [4] were the only ones to use antiangiogenic agents associated with chemotherapy and, at the time of the publication, had achieved more than 12 months' survival in one of their patients. However, due to the disparity of chemotherapeutic regimens utilized, drug choice must be individually tailored.

On the subject of pelvic irradiation, the literature remains inconclusive. In Table 1, it is shown that $40 \%(n=2 / 5)$ of patients with pelvic irradiation were still alive 6 months after publication of the case report, compared to $70 \%(n=10)$ of those without irradiation. Based on these data, pelvic irradiation does not appear to offer any benefits.

Metastases of uterine angiosarcomas are poorly described. Mendez et al. [14] and Medina et al. [15] have both reported lung and brain metastases, and one case of gingival metastasis was recorded by Medina et al. [15]. In their opinion, surgical excision was necessary to improve the patient's quality of life. In our case, the appearance of gingival and pulmonary metastases during chemotherapy could have worsened the prognosis, but the patient survived more than 4 years after treatment. Again, this may have been due to the chemotherapeutic regimen, but further studies are required to confirm this.

Cardiac metastasis from primary uterine angiosarcoma has never before been reported. In the case we describe here, development of the lesion suggested an aggressive neoplasm, but this was not borne out by the 53 months of progression-free survival at the time of cardiac surgery. Surgery was decided upon because of the 4-year survival of the patient in an attempt to prevent fatal heart failure or sudden death by pulmonary embolization or acute valvular obstruction. In the literature, four cases of successful excision of intracavitary extension to the heart from recurrent low-grade endometrial stromal sarcomas have been documented [16-19], and the authors considered the surgical approach to be a viable option. Unfortunately, in our case, the patient died postoperatively of acute rightsided cardiac failure. This risk has to be weighed against the risk of sudden death in untreated cases.

\section{Conclusion}

Here, we present the longest progression-free survival of primary uterine sarcoma treated by total abdominal hysterectomy with bilateral salpingo-oophorectomy achieved to date. Adjuvant chemotherapy seems to offer some benefit in terms of overall survival, but further studies are needed to determine the optimal regimen. Pelvic radiotherapy does not appear to improve overall survival. Limited distant metastases may be surgically resected in selected cases in order to improve quality of life or to prevent sudden death in untreated patients [19].

Conflict of interest There is no actual or potential conflict of interest in relation to this article.

\section{References}

1. Klob: Cited by Horgan E (1930) Hemangioma of uterus. Surg Gynecol Obstet 50:990

2. Rao J, Dekoven JG, Beatty JD, Jones G (2003) Cutaneous angiosarcoma as a delayed complication of radiation therapy for carcinoma of the breast. J Am Acad Dermatol 49:532-538

3. Abrahamson TG, Stone MS, Piette WW (2001) Cutaneous angiosarcoma. Adv Dermatol 17:279-299

4. Olawaiye AB, Morgan JA, Goodman A, Fuller AF, Penson RT (2008) Epithelioid angiosarcoma of the uterus: a review of management. Arch Gynecol Obstet 278:401-404

5. Cardinale L, Mirra M, Galli C, Goldblem JR, Pizzolito S, Falconeri G (2008) Angiosarcoma of the uterus: report of 2 new cases with deviant clinicopathologic features and review of the literature. Ann Diagn Pathol 12:217-221

6. Gatta G, Ciccolallo L, Kunkler I, Capocaccia R, Berrino F, Coleman M, De Angelis R, Faivre J, Lutz JM, Martinez C, Möller T, Sankila R, the EUROCARE Working Group (2006) Survival from rare cancer in adults: a population-based study. Lancet Oncol $7: 132-140$

7. Witkin GB, Askin FB, Geratz JD, Reddick RL (1987) Angiosarcoma of the uterus: a light microscopic, immunohistochemical, and ultrastructural study. Int J Gynecol Pathol 6:176-184

8. Milne DS, Hinshaw K, Malcolm AJ, Hilton P (1990) Primary angiosarcoma of the uterus: a case report. Histopathology 16:203-205

9. Quinonez GE, Paraskevas MP, Diocee MS, Lorimer SM (1991) Angiosarcomas of the uterus: a case report. Am J Obstet Gynecol 164:90-92 
10. Tallini G, Price FV, Carcangiu ML (1993) Epithelioid angiosarcoma arising in uterine leiomyomas. Am J Obstet Gynecol 100:514-518

11. Morrel B, Mulder AF, Chadha S, Tjokrowardojo AJ, Wijnen JA (1993) Angiosarcoma of the uterus following radiotherapy for squamous cell carcinoma of the cervix. Eur J Obstet Gynecol Reprod Biol 49:194-197

12. Schammel DP, Tavassoli FA (1998) Uterine angiosarcomas: a morphologic and immunohistochemical study of four cases. Am J Surgic Pathol 22:246-250

13. Darchenberg CB, Faust FJ, Borkowski A, Papadimitriuo JC (1994) Epithelioid angiosarcoma of the uterus arising in a leiomyoma with associated ovarian and tubal angiomatosis. Am J Clin Pathol 102:338-339

14. Mendez LE, Joy S, Angioli R, Estape R, Penalver M (1999) Primary uterine angiosarcoma. Gynecol Oncol 75:272-276
15. Medina BR, Barba EM, Torres AV, Trujillo SM (2001) Gingival metastasis as first sign of a primary uterine angiosarcoma. J Oral Maxillofac Surg 59:476-481

16. Vargas-Narron J, Keirn C, Barragan-Garcia R, Beltran-Ortega A, Rotberg T, Santana-Gonzalez A et al (1990) Intracardia extension of malignant uterine tumors. J Thorac Cardiovasc Surg 99:1099-1103

17. Mikami Y, Demopoulos RI, Boctor F, Febre EF, Harris M, Kronzen I et al (1999) Low-grade endometrial stromal sarcoma with intracardiac extension. Pathol Res Pract 195:501-508

18. Val-Bernarl JF, Hernandez-Nieto E (1999) Symptomatic intracavitary (non-invasive) cardiac metastasis from low-grade endometrial stromal sarcoma of the uterus. Pathol Res Pract 195:717-722

19. Yokoyama Y, OnoY ST, Fukuda I, Mizunuma H (2004) Asymptomatic intracardiac metastasis from a low-grade endometrial stromal sarcoma with successful surgical resection. Gynecol Oncol 92:999-1001 